\title{
Evaluation of the Relationship between Mandibular Third Molar and Mandibular Canal by Different Algorithms of Cone-beam Computed Tomography
}

\author{
${ }^{1}$ Mojdeh Mehdizadeh, ${ }^{2}$ Navid Ahmadi, ${ }^{3}$ Mahsa Jamshidi
}

\begin{abstract}
Objectives: Exact location of the inferior alveolar nerve (IAN) bundle is very important. The aim of this study is to evaluate the relationship between the mandibular third molar and the mandibular canal by cone-beam computed tomography.

Study design: This was a cross-sectional study with convenience sampling. 94 mandibular CBCTs performed with CSANEX 3D machine (Soredex, Finland) and 3D system chosen. Vertical and horizontal relationship between the mandibular canal and the third molar depicted by 3D, panoramic reformat view of CBCT and cross-sectional view. Cross-sectional view was our gold standard and other view evaluated by it.
\end{abstract}

Results: There were significant differences between the vertical and horizontal relation of nerve and tooth in all views $(p<0.001)$.

Conclusion: The results showed differences in the position of the inferior alveolar nerve with different views of $\mathrm{CBCT}$, so CBCT images are not quite reliable and have possibility of error.

Keywords: Cone-beam computed tomography, Inferior alveolar nerve bundle, Mandibular canal, Mandibular third molar, Reliable.

How to cite this article: Mehdizadeh M, Ahmadi N, Jamshidi M. Evaluation of the Relationship between Mandibular Third Molar and Mandibular Canal by Different Algorithms of Cone-beam Computed Tomography. J Contemp Dent Pract 2014;15(6): 740-745.

Source of support: Nil

Conflict of interest: None

\footnotetext{
${ }^{1}$ Associate Professor, ${ }^{2}$ Student, ${ }^{3}$ Assistant Professor

${ }^{1}$ Department of Oral and Maxillofacial Radiology, School of Dentistry, Torabinejad Dental Research Center; Dental Implants Research Center, Isfahan University of Medical Sciences, Isfahan, Iran

${ }^{2}$ Department of Dentistry, Isfahan University of Medical Sciences, Isfahan, Iran

${ }^{3}$ Department of Oral and Maxillofacial Radiology, School of Dentistry, Isfahan University of Medical Sciences, Isfahan, Iran

Corresponding Author: Navid Ahmadi, Student Isfahan Department of Dentistry, University of Medical Sciences Isfahan, Iran, Phone: +989131093769, e-mail: navid. ahmadi03@gmail.com
}

\section{INTRODUCTION}

Knowledge of exact location of the inferior alveolar nerve (IAN) bundle is a very important factor since this data reveals the regions where safe and quick removal of bone is possible and danger zones where special care must be used. ${ }^{1}$

The open extraction of third molars is one of the most commonly used procedures in dentoalveolar surgery, and after the surgery several complications can occur, ${ }^{2}$ therefore, location of the IAN in the surgical field is important because it can improve the reduction of complications, surgical planning, and quality of care. ${ }^{3}$

A preoperative evaluation of the exact location of the IAN and the roots of the mandibular third molar would help avoiding sensory deficiencies. ${ }^{4}$

For surgical extraction of Impacted Teeth, a true radiographic examination is needed to determine the adjacent anatomical structures. The proximity of the tooth to a cortex (buccal or lingual) will determine the procedure the surgeon will take. The region must be assessed for proximity to nerve and vessels. ${ }^{5}$

Several imaging approaches have been used to evaluate the site of the Mandibular canal, including panoramic radiography, ${ }^{6-9}$ computed tomography $(\mathrm{CT}),{ }^{8-10}$ conventional tomography, ${ }^{11}$ and the most recent one; cone-beam computed tomography (CBCT), ${ }^{9,12-13}$

Before CBCT was introduced, surgeons had only an estimate of the vertical position of the mandibular canal to the mandibular border. ${ }^{14}$

After the introduction of $\mathrm{CBCT}$, three-dimensional imaging became more readily available in order to use in dental applications. ${ }^{15}$

Cone-beam computed tomography images are reconstructed in a three-dimensional (3D) dataset with modification of the original cone-beam algorithm developed by Feldkamp et al. ${ }^{16} \mathrm{CBCT}$ is a medical image modality on the basis of a cone-shaped X-ray beam centered on a two-dimensional (2D) detector. The source-detector system rotates around the object producing a series of 2D images. ${ }^{16}$ Demand for CBCT in the oromaxillofacial region is increasing. ${ }^{17}$ 
Good aspects of CBCT imaging are its accessibility, easy handling and that it offers a real-size dataset with multi planar cross-sectional and 3D reconstructions. ${ }^{18-20}$

Cone-beam computed tomography can determine the exact location of the impacted teeth and their relationship to surrounding structures. ${ }^{21}$ Data gathered from imaging can be reformatted to show sequential slices through the oral and maxillofacial complex in the axial, coronal and sagittal planes. These data can be manipulated to produce precise $3 \mathrm{D}$ reconstructions of the area that surgeon wants to be evaluated, helps him find a clear picture of the exact location of the teeth in question. ${ }^{5}$

These scans allow the surgeon to gain an under-standing of the precise relationship between the mandibular third molar and the inferior alveolar canal. This increased understanding of the anatomical relationships of a modified surgical approach may be necessary to remove teeth or replacement allows the surgeon to reduce the risk of surgical planning. ${ }^{22}$

Cone-beam computed tomography is able to show the exact location of the nerve enough to predict binding to the proximal nerve during surgery, so it is possible to modify the method or to choose a different approach as an alternative to surgery in high-risk procedures. ${ }^{3}$

Vannier said that the recent developments in the synthesis and optimization of CBCT reconstruction algorithms allows to exploit the full potential of the detectors in $\mathrm{CBCT}, \mathrm{CBCT}$ will provide important advantages for craniofacial imaging. It is expected that advances in cone beam reconstruction algorithms and post processing reduce this problem. ${ }^{11,23}$

Yabroudi and colleagues ${ }^{15}$ studied on forty-seven mandibular third molars in 30 patients who were in close contact with mandibular canal in panoramic radiographs. In this study, 3D relationship between the mandibular third molar and the mandibular canal in cone-beam computed tomography were examined. The results of this study showed that from forty-seven of wisdom teeth were close to mandibular canal, 8 mandibular canals were buccal of third molars, eleven of them were lingual to third molars and the 7 of them were between the roots. $\mathrm{CBCT}$ in demonstration of existence or absence of cortical bone between nerve and roots was more accurate than in panoramic radiographs. So, it can be used for risk assessment of surgical procedures.

Bell et al reported that the mean of sensitivity and specificity of panoramic images in predicting exposure were of 66 and $74 \%$ respectively. They reported that these values vary between observers and emphasized the need for greater standardization of diagnostic criteria. ${ }^{24}$

Angelopoulus et $\mathrm{al}^{6}$ were compared digital and conventional panoramic radiography and $\mathrm{CBCT}$ for detecting mandibular canal in different regions of the mandible. CBCT compared to other methods for such a task is found better. The posterior third of the mandibular canal that was the best visualization of the entire procedure was followed intermediate.

Lofthag-Hansen et $\mathrm{al}^{3}$ evaluated the mandibular canal and alveolar ridge distance by cross-sectional view of CBCT images. In this study, visibility of alveolar ridge crest was considered superior than mandibular canal. Observers only marked mandibular canals clearly in one third of cases with one predetermined cross-sectional image, but by more images visibility of mandibular canal was increased. This result points to the importance of each successive image available to improve the localization of the mandibular canal.

The aim of this study is to evaluate the relationship between the mandibular third molar and the mandibular canal by cone-beam computed tomography with elimination of hard tissue.

\section{MATERIALS AND METHODS}

In this cross-sectional study, 94 mandibular CBCTs performed with CSANEX 3D machine (Soredex, Finland) and 3D system from patients who had near relationship between mandibular canal and mandibular third molar in panoramic radiography were simply chosen by a radiologist, and those pictures that were without superimposition excluded from study.

Pictures reconstructed by 3D (Fig. 1) panoramic reformat view of CBCT (Fig. 2) and cross-sectional image views of $\mathrm{CBCT}$ (Fig. 3) and a radiologist separately evaluated the vertical and horizontal position of the mandibular nerve and the mandibular third molars. For standardization of conditions, evaluation was designed at certain hours of the day in a room with a computer specifically to room lighting conditions and contrast on the screen does not affect the measurement accuracy.

Since in cross-sectional view of CBCT the images are multiple and possible error detection status is minimized, Gold standard in this study was cross-sectional view of CBCT and other images were compared with that. ${ }^{13}$

Vertical relationship between the mandibular third molar root and the mandibular canal classified into three categories according to the position of the tip of the root:

- The root apex at the upper half of the mandibular canal.

- The root apex at the lower half of the mandibular canal.

- The root apex under the inferior wall of the mandibular canal.

The horizontal relationship between the mandibular canal and the mandibular third molar classified in terms of the position into one of the four following categories: 


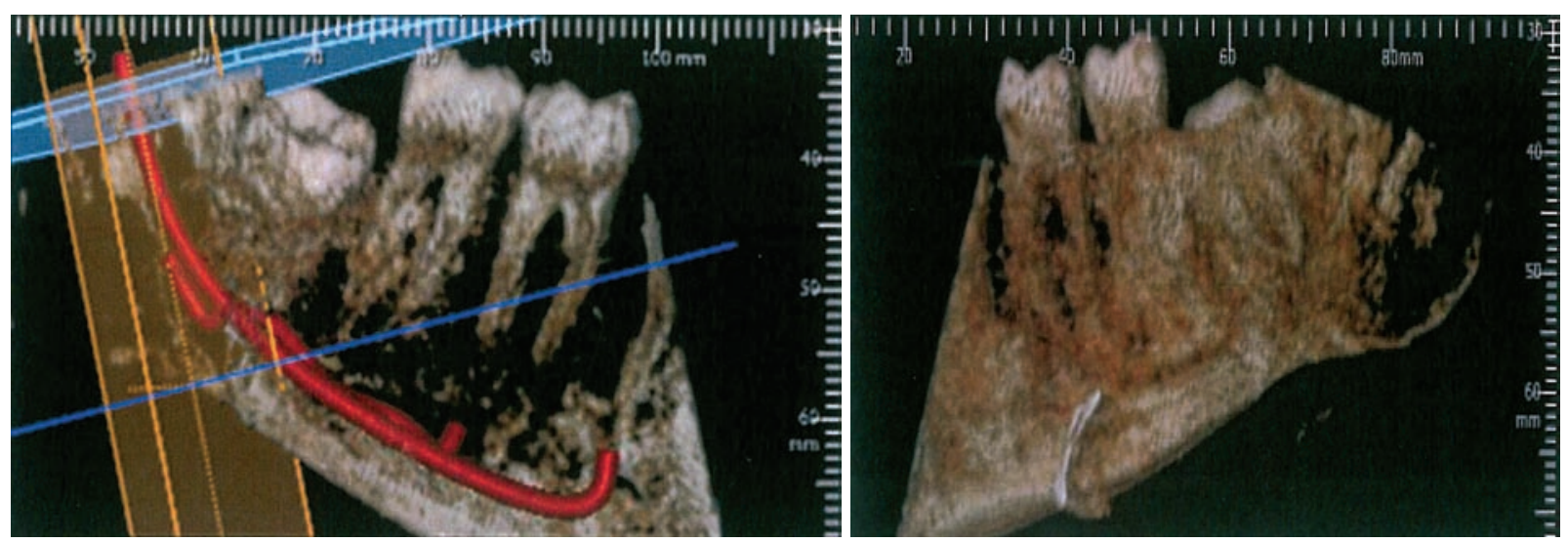

Fig. 1: Three dimensional view of cone-beam computed tomography

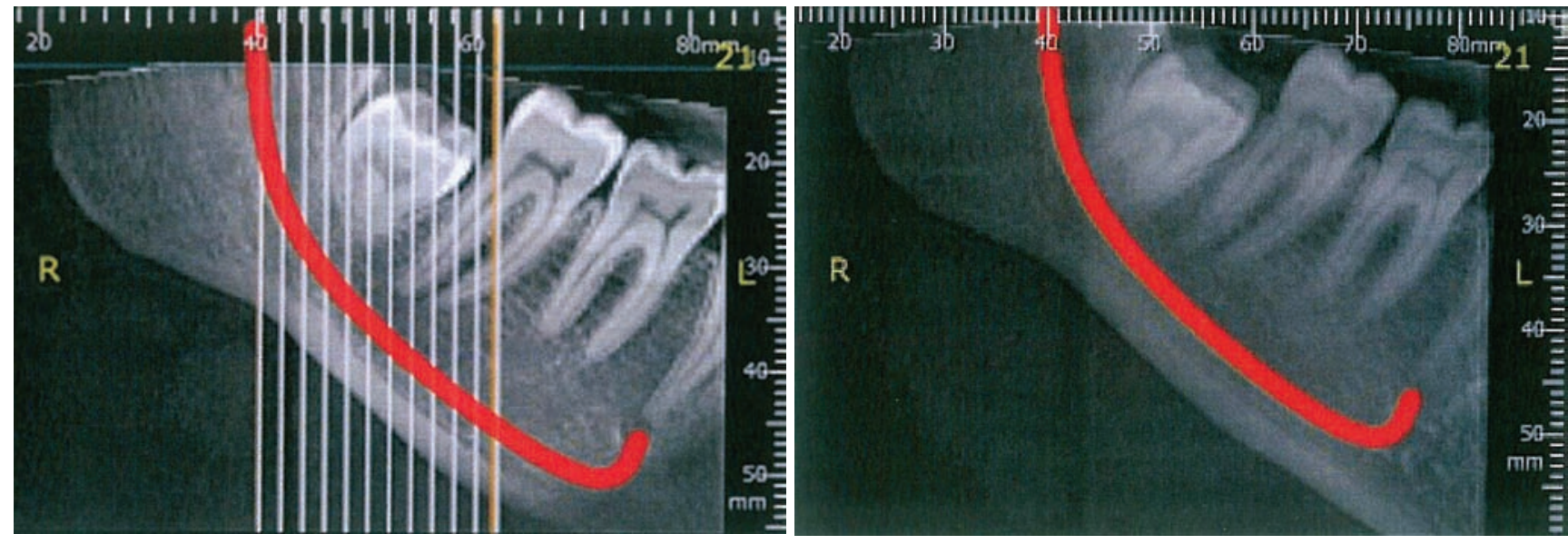

Fig. 2: Panoramic reformat view of cone-beam computed tomography

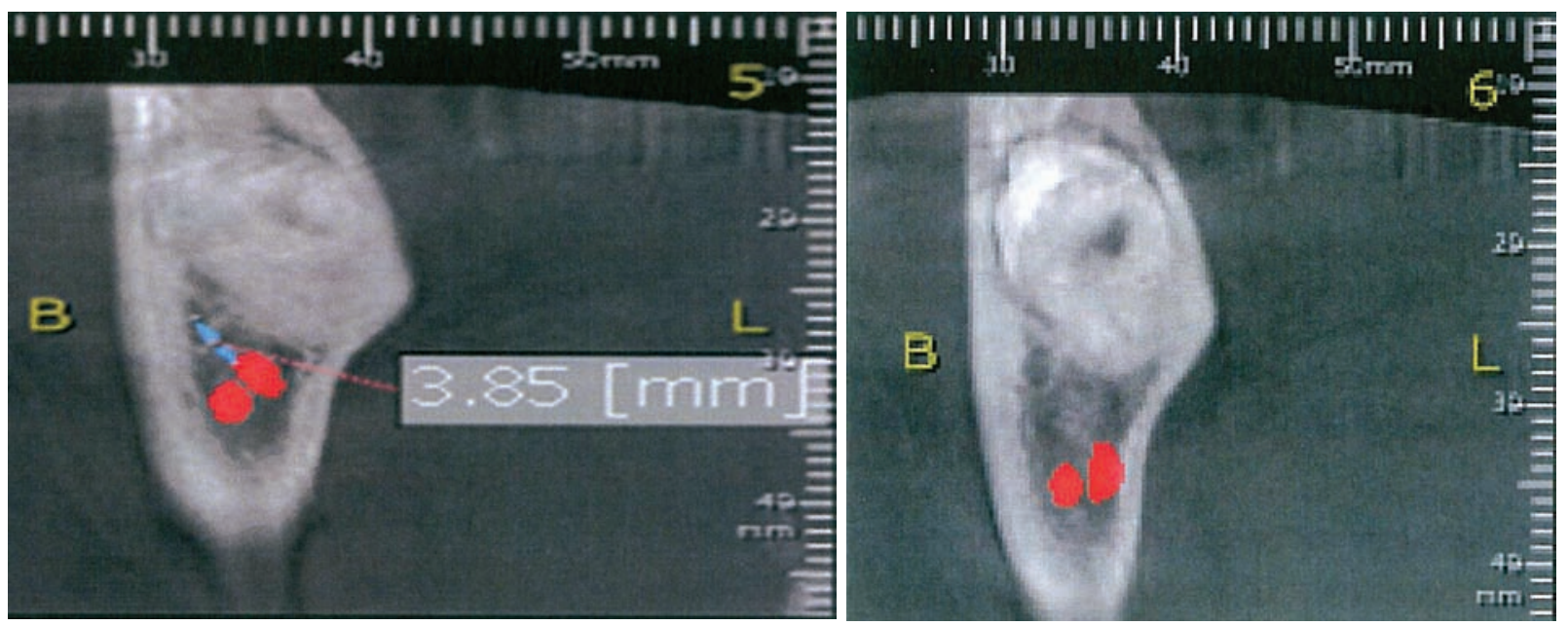

Fig. 3: Cross-sectional view of cone-beam computed tomography

- Roots were located at the buccal of nerve.

- Roots were located at the inferior of nerve.

- Roots were located at the lingual of nerve.

- Nerve was located between the roots.

Horizontal and vertical relations between nerves and teeth in each view separately recorded and the data were entered and analyzed in software SPSS. ${ }^{20}$ Kappa ratio and chi-square test was used for statistical analysis. Kappa ratio was considered between 0.1 and 0.9 and significance level was $\alpha=0.05$.

\section{RESULTS}

In this study, 94 patients with low alveolar canal and wisdom teeth distance on the CBCT images had been studied. Images using three views: cross-sectional, panoramic reformat view of CBCT and 3D were analyzed, cross-sectional view taken as the standard view and the images were compared with it. Figure 4 is an example of injury to the inferior alveolar nerve in all three views.

Tables 1 and 2 compare the results of different view in vertical and horizontal relationship. 

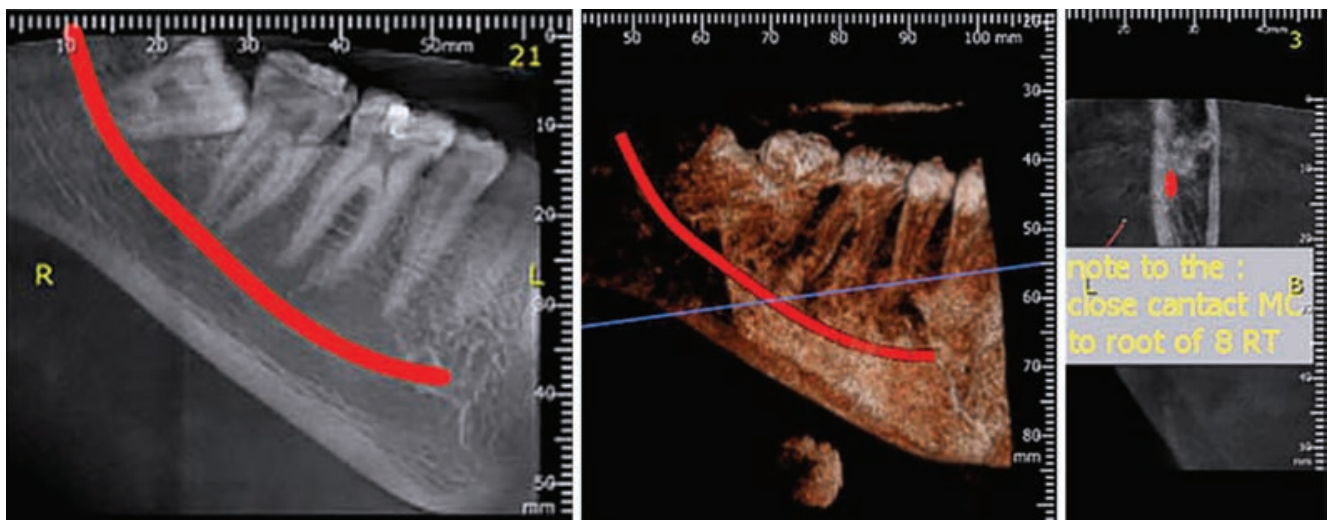

Fig. 4: Injury to the inferior alveolar nerve

Table 1: The results of different view in vertical relationship

\begin{tabular}{|c|c|c|c|c|c|c|}
\hline & \multicolumn{2}{|c|}{ Cross-sectional view } & \multicolumn{2}{|c|}{$3 D$ view } & \multicolumn{2}{|c|}{ Panoramic reformat view of $C B C T$} \\
\hline & Frequency & Percentage (\%) & Frequency & Percentage (\%) & Frequency & Percentage (\%) \\
\hline Root apex at the upper half of the canal & 89 & 93.7 & 88 & 92.6 & 85 & 89.5 \\
\hline Root apex at the lower half of the canal & 4 & 4.2 & 5 & 5.3 & 8 & 8.4 \\
\hline Root apex under the canal & 1 & 1.1 & 1 & 1.1 & 1 & 1.1 \\
\hline
\end{tabular}

CBCT: Cone-beam computed tomography; 3D: Three-dimensional

Table 2: The results of different view in horizontal relationship

\begin{tabular}{|c|c|c|c|c|c|c|}
\hline & \multicolumn{2}{|c|}{ Cross-sectional view } & \multicolumn{2}{|c|}{$3 D$ view } & \multicolumn{2}{|c|}{ Panoramic reformat view of $C B C T$} \\
\hline & Frequency & Percentage (\%) & Frequency & Percentage (\%) & Frequency & Percentage (\%) \\
\hline Root apex at the buccal of the canal & 13 & 13.7 & 6 & 6.3 & 3 & 3.2 \\
\hline Root apex at the lingual half of the canal & 64 & 67.4 & 82 & 86.3 & 87 & 91.6 \\
\hline Root apex inferior the canal & 17 & 17.9 & 6 & 6.3 & 4 & 4.2 \\
\hline
\end{tabular}

CBCT: Cone-beam computed tomography; 3D: Three-dimensional

Kappa ratio for comparison of the vertical distances between 3D view and cross-sectional view was 0.713 and $\mathrm{p}$-value was $<0.001$ that chi-square test approved it.

Kappa ratio for comparison of the vertical distance between panoramic reformat view of CBCT and crosssectional view was 0.696 and $p$-value was $<0.001$ that chi-square test approved it.

Kappa ratio for comparison of the horizontal distances between 3D view and cross-sectional view was 0.283 and p-value was $<0.001$ that chi-square test approved it.

Kappa ratio for comparison of the horizontal distance between panoramic reformat view of CBCT and crosssectional view was 0.197 and p-value was 0.001 that chisquare test approved it.

\section{DISCUSSION}

Detecting of the exact location of the inferior alveolar nerve is very important to determine the safe location of bone removal. ${ }^{1}$

Third molar surgery is the most common cause of injury to the inferior alveolar nerve, ${ }^{2}$ so the knowledge of the exact location of the nerve can reduce surgical complications. ${ }^{3}$

If you decide to make a third molar surgery the radiographic examination of the teeth and surrounding structures is required. ${ }^{5}$
There are various methods of imaging for evaluating mandibular canal locations, including: panoramic, conventional tomography, computed tomography and CBCT. $^{6-9}$

Introduction of new radiographic techniques is enabled to see anatomical structures in all aspects without superimposition. ${ }^{25}$

Before the accessibility of CBCT surgeons just could determine the vertical position of the mandibular canal in regard to mandibular border. ${ }^{15}$

The picture quality and contrast between adjacent structures are important factors in the detection of anatomical structures on a radiograph. ${ }^{10}$

The use of 2D images to view 3D structures can be difficult to determine the location of the tooth impaction. The old solution to this problem was using two techniques to prepare radiology SLOB or perpendicular, respectively. ${ }^{26}$

CBCT 3D images are helpful in determining the exact location of mandibular canal. ${ }^{16}$

Vannier et al in a study on new techniques of CBCT images reconstruction showed that the new method has several advantages in imaging of craniofacial and can reduce surgical complications. ${ }^{11,23}$

Bell et al reported that the sensitivity and specificity of panoramic images in predicting exposure were 66 
and $74 \%$ on average respectively. They also reported that these values varied widely among observers and emphasized the need for further standardization of diagnostic criteria. ${ }^{24}$

The results are consistent with the results of Bell et al, in this study, it is not possible to determine the exact position of the nerve using other methods such as surgery, so the results has been influenced by the individual observer.

Angelopoulus et al in a study that compared analog and digital panoramic and CBCT images in the inferior alveolar place, showed that CBCT is superior to other radiography in the determining the exact location of inferior alveolar canal. ${ }^{6}$

Yabroudi et al studied on 47 third molars in 30 patients who were in close proximity to alveolar nerve. In this study, dental panoramic and CBCT images were obtained. Results of this study showed that from 47 teeth adjacent to nerve, 8 of them were at the buccal of the teeth, 11 of them were at lingual of the teeth, 21 of them were inferior of the teeth and 7 of them were between the roots of the third molar teeth. The results of this study showed that CBCT is more accurate than panoramic images in determining the location of the nerve. ${ }^{15}$

In the present study, among the 94 samples with close proximity to the nerve, 89 samples were in the upper half of the mandibular canal, four samples were in the lower half of the mandibular canal and one sample was placed under the canal.

From the horizontal aspect 13 canals were at lingual of the teeth, 64 canals were at the buccal of the teeth and 17 of them were inferior the roots and no canal was seen between the roots.

Hansen et al evaluated the place of alveolar crest and the mandibular canal in CBCT cross-sectional images. The results showed that observation of alveolar crest is easier than the mandibular canal. In this study, only one-third of the mandibular canal was easily visible. The results of this study also showed that using the images with more sections is helpful for determining the exact location of mandibular canal. ${ }^{13}$

In this study, which examines the three views (panoramic reformat view of $\mathrm{CBCT}$, cross-sectional and 3D) of CBCT radiography, cross-sectional view was considered as the gold standard for determining the position of the nerve-tooth relation.

In this study, the differences between the three views in the vertical and horizontal distance was statistically significant ( $p<0.05$ ), so the results of this study is the same with the Hansen et al's study which mentioned that more sections is helpful to determine the exact location of mandibular canal.
In the present study, all of the views showed the same results in position that the root was under the nerve, but a little differences between 3D and cross-sectional and panoramic reformat view of CBCT and cross-sectional were seen that in majority of them nerve position was lower than the exact position in cross-sectional view (Tables 1 and 2).

Chi-square test revealed significant difference $(p<0.05)$ in the vertical position of the nerve in relation to the three views of the CBCT, therefore, it can be said that the vertical relationship between the nerve and the tooth cannot be determined only by one view of radiographs and using of other views can be helpful to determine the exact location of mandibular canal.

In this study, there were some differences between the $3 \mathrm{D}$ and panoramic reformat view of CBCT with cross-sectional view in horizontal relationship between the mandibular canal and the roots of the teeth. The most differences were that 3D and panoramic reformat view of CBCT which showed that the nerve is at the buccal of the roots but the exact location of nerve is under the canal or at lingual by the cross-sectional view.

Chi-square test revealed significant difference $(p<0.05)$ in the horizontal position of the nerve in relation to the three views of the $\mathrm{CBCT}$, so it can be said that the horizontal relationship between the nerve and the tooth cannot be determined only by one view of radiographs and using of other views can be helpful to determine the exact location of mandibular canal.

\section{CONCLUSION}

The results of this study showed differences in the position of the inferior alveolar nerve with different views of CBCT. The results of this study showed that CBCT images are not quite reliable and have possibility of error.

So using of more sections and different views can be useful to determine the exact location of mandibular canal.

\section{REFERENCES}

1. Eyrich G, Seifert B, Matthews F, Matthiessen U, Heusser $\mathrm{CK}$, Kruse AL, et al. 3-Dimensional imaging for lower third molars: is there an implication for surgical removal? J Oral Max Surg 2011;69(7):1867-1872.

2. Jung Y-H, Nah K-S, Cho B-H. Correlation of panoramic radiographs and cone beam computed tomography in the assessment of a superimposed relationship between the mandibular canal and impacted third molars. Imaging science in dentistry 2012;42(3):121-127.

3. Agbaje JO, Sun Y, De Munter S, Schepers S, Vrielinck L, Lambrichts I, et al. CBCT-based predictability of attachment of the neurovascular bundle to the proximal segment of the mandible during sagittal split osteotomy. Int J Oral Max Surg 2012. 
4. Flygare L, Öhman A. Preoperative imaging procedures for lower wisdom teeth removal. Clin Oral Invest 2008;12(4): 291-302.

5. Deepak C, Saravanan B, Kumar SK. CBCT-A Paradigm Shift in the Management of Dental Impactions. Indian J Multi Dentist 2011;1(2):68.

6. Angelopoulos C, Thomas S, Hechler S, Parissis N, Hlavacek $\mathrm{M}$. Comparison between digital panoramic radiography and cone-beam computed tomography for the identification of the mandibular canal as part of presurgical dental implant assessment. J Ora Max Surg 2008;66(10):2130-2135.

7. Lindh C, Petersson A. Radiologic examination for location of the mandibular canal: a comparison between panoramic radiography and conventional tomography. Int J Oral Max Imp 1989;4(3):249.

8. Naitoh M, Katsumata A, Kubota Y, Hayashi M, Ariji E. Relationship between cancellous bone density and mandibular canal depiction. Imp Dent 2009;18(2):112-118.

9. Tantanapornkul W, Okouchi K, Fujiwara Y, Yamashiro M, Maruoka Y, Ohbayashi N, et al. A comparative study of conebeam computed tomography and conventional panoramic radiography in assessing the topographic relationship between the mandibular canal and impacted third molars. Oral Surg Oral Med Oral Path Oral Rad Endod 2007;103(2):253.

10. Lou L, Lagravere MO, Compton S, Major PW, Flores-Mir C. Accuracy of measurements and reliability of landmark identification with computed tomography (CT) techniques in the maxillofacial area: a systematic review. Oral Surg Oral Med Oral Path Oral Rad Endod 2007;104(3):402-411.

11. Vannier M. Craniofacial computed tomography scanning: technology, applications and future trends. Orthod Cranio Research 2003;6(s1):23-30.

12. Kamburoğlu K, Kılıç C, Özen T, Yüksel SP. Measurements of mandibular canal region obtained by cone-beam computed tomography: a cadaveric study. Oral Surg Oral Med Oral Path Oral Rad Endod 2009;107(2):e34-e42.

13. Lofthag-Hansen S, Gröndahl K, Ekestubbe A. Cone-Beam CT for Preoperative Implant Planning in the Posterior Mandible: Visibility of Anatomic Landmarks. Clin Imp Dent Related Research 2009;11(3):246-255.

14. Jhamb A, Dolas RS, Pandilwar PK, Mohanty S. Comparative efficacy of spiral computed tomography and orthopantomography in preoperative detection of relation of inferior alveolar neurovascular bundle to the impacted mandibular third molar. J Ora Max Surg 2009;67(1):58-66.
15. Yabroudi-BDS F. Cone beam tomography (CBCT) as a diagnostic tool to assess the relationship between the inferior alveolar nerve and roots of mandibular wisdom teeth.

16. Feldkamp L, Davis L, Kress J. Practical cone-beam algorithm. JOSA A 1984;1(6):612-619.

17. De Vos W, Casselman J, Swennen G. Cone-beam computerized tomography $(\mathrm{CBCT})$ imaging of the oral and maxillofacial region: a systematic review of the literature. Int J Oral Max Surg 2009;38(6):609-625.

18. Kipp DP, Goldstein BH, Weiss WW. Dysesthesia after mandibular third molar surgery: a retrospective study and analysis of 1,377 surgical procedures. J Amer Dent Asso 1980;100(2):185-192.

19. Libersa P, Savignat M, Tonnel A. Neurosensory disturbances of the inferior alveolar nerve: a retrospective study of complaints in a 10-year period. J Ora Max Surg 2007;65(8):1486-1489.

20. Monaco G, Montevecchi M, Bonetti GA, Gatto MRA, Checchi L. Reliability of panoramic radiography in evaluating the topographic relationship between the mandibular canal and impacted third molars. J Amer Dent Asso 2004;135(3): 312-318.

21. Cevidanes LH, Styner MA, Proffit WR. Image analysis and superimposition of 3-dimensional cone-beam computed tomography models. American journal of orthodontics and dentofacial orthopedics: official publication of the American Association of Orthodontists, its constituent societies, and the American Board of Orthodontics 2006;129(5):611.

22. Umar G, Bryant C, Obisesan O, Rood J. Correlation of the radiological predictive factors of inferior alveolar nerve injury with cone beam computed tomography findings. Oral Surg 2010;3(3):72-82.

23. Swennen GR, Schutyser F. Three-dimensional cephalometry: spiral multi-slice vs cone-beam computed tomography. Amer J Orthod Dent Orthoped 2006;130(3):410-416.

24. Bell GW, Rodgers JM, Grime RJ, Edwards KL, Hahn MR, Dorman ML, et al. The accuracy of dental panoramic tomographs in determining the root morphology of mandibular third molar teeth before surgery. Oral Surg Oral Med Oral Path Oral Rad Endod 2003;95(1):119-125.

25. Oliveira-Santos C, Capelozza ALÁ, Dezzoti MSG, Fischer CM, Poleti ML, Rubira-Bullen IRF. Visibility of the mandibular canal on CBCT crosssectional images. J App Oral Sci 2011;19(3):240-243.

26. White SC, Pharoah MJ. Oral radiology: principles and interpretation: Mosby; 2008. 